\title{
Identifying efficient natural bioreductants for the preparation of graphene and graphene-metal nanoparticle hybrids with enhanced catalytic activity from graphite oxide
}

M.J. Fernández-Merino, S. Villar-Rodil*, J.I. Paredes, P. Solís-Fernández, L. Guardia, R. García, A. Martínez-Alonso, J.M.D. Tascón

Instituto Nacional del Carbón, INCAR-CSIC, Apartado 73, 33080 Oviedo, Spain

\begin{abstract}
The implementation of green approaches towards the preparation of graphene and graphene-based materials with enhanced functionality from graphite oxide has been relatively little explored. Particularly, the use of bioreductants and the testing of their relative efficacies is an incipient area of research. Here, a pool of 20 environmentally friendly, natural antioxidants has been tested for their ability to reduce graphene oxide. These antioxidants were mostly vitamins, amino acids and organic acids. By establishing a protocol to systematically compare and optimize their performance, several new efficient bioreductants of graphene oxide have been identified, namely, pyridoxine and pyridoxamine (vitamin $\mathrm{B}_{6}$ ), riboflavin (vitamin $\mathrm{B}_{2}$ ), as well as the amino acids arginine, histidine and tryptophan. These biomolecules were used to prepare reduced graphene oxide-silver nanoparticle hybrids that displayed colloidal stability in water in the absence of additional dispersants. Particularly, hybrids prepared with pyridoxamine exhibited a combination of long-term colloidal stability and exceptionally
\end{abstract}

\footnotetext{
* Corresponding author: Telephone number: (+34) 9851190 90. Fax number: (+34) 9852976 62. E-mail address: silvia@incar.csic.es (S. Villar Rodil)
} 
high catalytic activity among silver nanoparticle-based catalysts in the reduction of $p$ nitrophenol with $\mathrm{NaBH}_{4}$. Thus, in addition to expanding substantially the number of green reductants available for graphene oxide reduction, the present results underline the idea that proper selection of bioreductant can be relevant to achieve graphene-based materials with improved performance.

\section{Introduction}

Since its first isolation in 2004, graphene has aroused a great deal of interest in the scientific community, and particularly in the materials research realm, given that its exceptional physical properties promise outstanding performance in a large number of potential applications [1-3]. However, an obvious prerequisite for the practical use of graphene is the availability of suitable methods that allow its mass production and processing. In this regard, one of the most promising and hitherto widely investigated approaches relies on the use of graphite oxide, which typically comprises the exfoliation of this strongly oxygenated derivative of graphite in aqueous or organic medium followed by chemical reduction of the resulting single-layer sheets (graphene oxide sheets) [4-6]. Hydrazine and some of its derivatives, and later on other reagents commonly used in organic chemistry for the reduction of oxygen-containing groups (e.g., $\mathrm{LiAlH}_{4}$ ), were found to be highly effective reductants for graphene oxide, and are still broadly employed today for this purpose [4-10]. Nevertheless, the high toxicity and environmental hazard of these reagents have recently driven a move towards the exploration of safer alternatives, to better comply with the principles of green chemistry and sustainability. Thus, solvothermal, electrochemical, catalytic [11] and 
photocatalytic [12-17] methods as well as the use of environmentally friendly reductants have been proposed during the last two years for the reduction of graphene oxide [18].

Vitamin $\mathrm{C}$ was the first green reducing agent that was reported to be effective in the deoxygenation of graphene oxide, affording dispersions of reduced graphene oxide (RGO) sheets in water and some organic solvents whose extent of reduction and colloidal stability matched those of dispersions obtained with hydrazine [19-22]. Such discovery suggested that other innocuous and abundant biomolecules could also act as efficient reductants in the preparation of RGO. Studies carried out subsequently confirmed indeed that certain saccharides (glucose, fructose and sucrose) and polysaccharides (dextran) [23-25],polyphenols (e.g., epigallocatechin gallate and related compounds present in tea solutions, or tannic acid) [26-28] and natural phenolic acids [29,30], plant extracts [31], a catecholamine polymer [poly(norepinephrine)] [32], the hormone melatonin [33,34], a tripeptide (L-gluthatione) [35], a protein [bovine serum albumin (BSA)] [36], and bacteria [37,38] could reduce graphene oxide.

However, the number of biomolecules available at present for the reduction of graphene oxide is still relatively limited, and having a wider range of effective bioreductants to call upon for specific purposes would be highly desirable. There is a large number of natural antioxidants, including amino acids, vitamins and organic acids whose reducing ability towards graphene oxide and potential role in enhancing the functionality of the resulting material is unknown and unexplored. It has been shown in some cases that the use of selected biomolecules lends added value to RGO other than just acting as reductants of the starting graphene oxide sheets. For example, dextran and tea polyphenols have been reported to increase the colloidal stability in water and/or biocompatibility of RGO [24,26]. However, other potential benefits of using reducing 
biomolecules in the preparation of graphene-based materials that could be relevant for applications have been, to the best of our knowledge, far less explored [32].

In the present work, (i) we identify a number of small biomolecules (amino acids and vitamins) as efficient bioreductants of grapheme oxide dispersions, and (ii) significantly, we demonstrate that proper selection of specific bioreductants affords graphene-based materials with enhanced performance towards relevant uses. First of all, a set of 20 biomolecules was investigated for their potential use in the reduction of aqueous graphene oxide dispersions. These biomolecules were mainly chosen on the basis of their known reducing and/or antioxidant activity in the biochemical context, although their reducing power towards graphene oxide was unknown. Several of the tested biomolecules were shown to be efficient reductants of graphene oxide. Then, the previously identified bioreductants were used for the preparation of RGO-silver nanoparticle (Ag NP) hybrids. Furthermore, the use of selected biomolecules was shown to afford hybrids that exhibited an attractive combination of long-term colloidal stability in water and exceptionally high catalytic activity, as gauged from the study of a model reaction (reduction of $p$-nitrophenol to $p$-aminophenol), making them ideal candidates for practical uses.

\section{Experimental section}

Unless otherwise specified, all the chemicals used were supplied by Sigma-Aldrich ${ }^{\circledR}$ Chemistry. 


\subsection{Preparation of graphene oxide}

Graphite oxide was obtained as thick slurry from natural graphite powder (Fluka 50870) by the Hummers method as described elsewhere [39]. Aqueous graphene oxide dispersions were prepared from the slurry through bath sonication (JP Selecta Ultrasons System, $40 \mathrm{KHz}$ ) for $4 \mathrm{~h}$ followed by centrifugation (Eppendorf 5424 microcentrifuge) at $10,000 \mathrm{~g}$ for $10 \mathrm{~min}$ to sediment the non-exfoliated graphite oxide particles. The supernatant, a stable dispersion mostly made up of individual, single-layer graphene oxide sheets, was collected and kept for further use. The concentration of such dispersion was determined by UV-vis absorption spectroscopy as described elsewhere [39].

\subsection{Reduction of aqueous graphene oxide dispersions with biomolecules}

To investigate and compare the performance of different biomolecules in the reduction of graphene oxide, aqueous dispersions of the latter at a concentration of $0.1 \mathrm{mg} \mathrm{mL}^{-1}$ and $\mathrm{pH}$ of $\sim 10$ (adjusted by the addition of $25 \%$ ammonia solution) were mixed with varying amounts of the biomolecules and heated to $95{ }^{\circ} \mathrm{C}$ for different periods of time. The following biomolecules were tested (see Table 1 for their chemical structures): pyridoxine and pyridoxamine dihydrochloride (different forms of vitamin $\mathrm{B}_{6}$ ), (-)riboflavin (vitamin $\mathrm{B}_{2}$ ) and riboflavin 5'-monophosphate salt hydrate (a more soluble form of the latter), citric acid, fumaric acid, L-malic acid, and L-tartaric acid, L-arginine, L-aspargine, L-carnosine, glycine, L-glutamic acid, L-gluthatione, L-histidine, Lmethionine, L-phenylalanine, L-tryptophan, L-tyrosine, and $\alpha$-D-glucose. The extent of reduction achieved with a given biomolecule, concentration of biomolecule and heating time was initially assessed through UV-vis absorption spectroscopy, and more specifically by measuring the position of the absorption peak corresponding to $\pi \rightarrow \pi^{*}$ 
transitions of $\mathrm{C}=\mathrm{C}$ bonds $\left(\lambda_{\max }\right)$ in graphene oxide [22]. Some of the tested biomolecules (and/or their oxidized counterparts) exhibited absorption features in the $\mathrm{UV}$-vis spectrum that prevented an accurate determination of $\lambda_{\max }$ for the reduced graphene oxide sheets (typically located between $\sim 231$ and $268 \mathrm{~nm}$ ). In these cases, the reduced dispersions were dialyzed (Spectra/Por ${ }^{\circledR}$ Float-A-Lyzer ${ }^{\circledR}$ G2 dialysis membrane made of cellulose ester with $10^{6}$ Da molecular weight cut-off, from Spectrum Laboratories) for the time required to remove the interfering species.

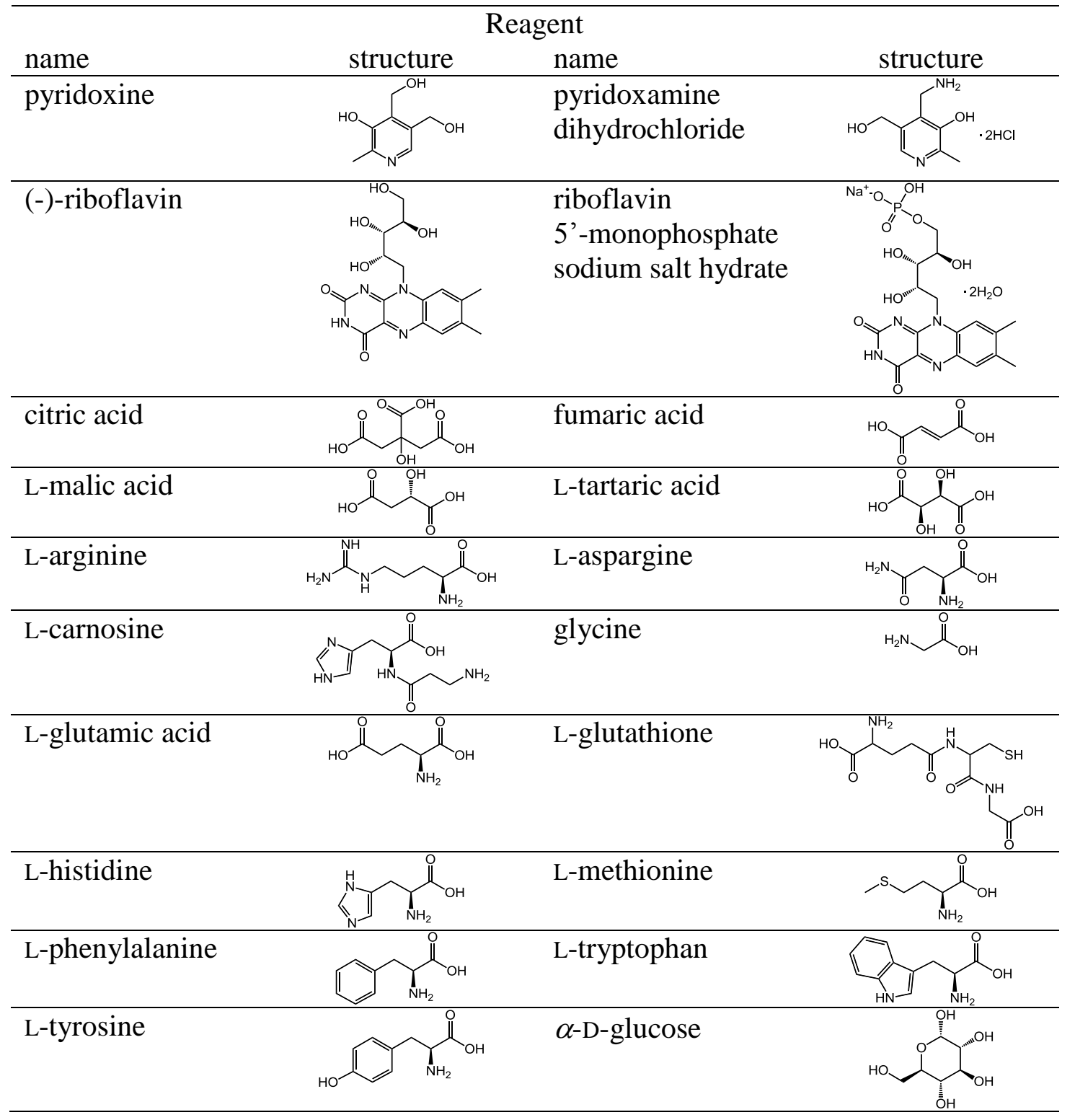


Table 1. Chemical structure of the biomolecules used in the study together with their names.

\subsection{Preparation of $R G O-A g N P$ hybrids}

The biomolecules that were found to be successful in the reduction of graphene oxide were also tested for simultaneous reduction of graphene oxide and a silver salt $\left(\mathrm{AgNO}_{3}\right)$ with the aim of preparing RGO-Ag NP hybrids. Aqueous solutions containing $0.1 \mathrm{mg}$ $\mathrm{mL}^{-1}$ graphene oxide, $0.34 \mathrm{mM} \mathrm{AgNO}_{3}$ and varying concentrations of bioreductant (from 0.125 to $8 \mathrm{mM}$, depending on the particular biomolecule) were heated at $95{ }^{\circ} \mathrm{C}$ for 8 hours. To eliminate possible residual unreacted silver salt and bioreductant (and/or its reacted, oxidized form), the obtained hybrids were subjected to several cycles of sedimentation by centrifugation (20000 $\mathrm{g}, 30 \mathrm{~min}$ ) and re-suspension in Milli-Q water.

\subsection{Catalytic activity of RGO-Ag NP hybrids}

The catalytic activity of selected RGO-Ag NP hybrids was evaluated towards reduction of $p$-nitrophenol to $p$-aminophenol with $\mathrm{NaBH}_{4}$ at $\mathrm{RT}$ in aqueous medium. The reaction progress was monitored by UV-vis absorption spectroscopy. To this end, equal volumes of freshly prepared aqueous solutions of $p$-nitrophenol $(10 \mathrm{mM})$ and $\mathrm{NaBH}_{4}(3 \mathrm{M})$ were mixed and stirred vigorously. The high molar ratio of $\mathrm{NaBH}_{4}$ to $p$-nitrophenol (300:1) ensured an excess amount of the former and hence that its concentration remained essentially constant during the reduction reaction. This molar ratio was determined by gradually increasing the relative amount of $\mathrm{NaBH}_{4}$ up to the point where further increments did not have any effect on the reaction kinetics. Then, $25 \mu \mathrm{L}$ of such mixture were added to $2.1 \mathrm{~mL}$ of an aqueous RGO-Ag NP dispersion whose concentration of $\mathrm{Ag}$ NPs had been adjusted to $\sim 3-4 \times 10^{9} \mathrm{~mL}^{-1}$. After vigorous shaking for a few seconds, the 
absorbance of the mixture at $400 \mathrm{~nm}$ was monitored at $0.25 \mathrm{~s}$ time intervals. Several consecutive reaction rounds were measured to determine the stability of the catalyst.

\subsection{Characterization techniques}

UV-vis absorption spectroscopy measurements were carried out with a double-beam He $\lambda$ ios $\alpha$ spectrophotometer (Thermo Spectronic). Thermogravimetric analysis (TGA) was performed by means of an SDT Q600 thermobalance (TA Instruments), using Pt crucibles, under $100 \mathrm{~mL} \mathrm{~min}^{-1} \mathrm{Ar}$ flow at a heating rate of $10{ }^{\circ} \mathrm{C} \mathrm{min}{ }^{-1}$. Attenuated total reflection Fourier transform infrared (ATR-FTIR) spectra were recorded on a Nicolet 3700 spectrometer (Thermo Scientific) using diamond as ATR crystal. X-ray photoelectron spectroscopy (XPS) measurements were carried out on a SPECS system under a pressure of $10^{-7} \mathrm{~Pa}$ with a monochromatic $\mathrm{Al} \mathrm{K} \mathrm{K}_{\alpha} \mathrm{X}$-ray source operated at 100 W. The surface elemental composition of the samples was determined from the integrated intensities of the main XPS peaks that were detected in survey spectra. To investigate the samples by TGA, ATR-FTIR spectroscopy and XPS, the dispersions were processed into free-standing paper-like films by vacuum filtration through alumina membrane filters $47 \mathrm{~mm}$ in diameter and $0.2 \mu \mathrm{m}$ of pore size (Whatman). The sheet resistance of the different paper-like films, cut into $10 \times 10 \mathrm{~mm}^{2}$ squares, was determined by the van der Pauw method with a home-made setup (Agilent 6614C DC power supply and Fluke 45 digital multimeter). Proper characterization of the RGO material itself by these techniques required thorough removal of the bioreductants and/or their oxidized products prior to the filtration step. Otherwise, contributions from these species to the spectra and thermograms of the paper-like films tended to obscure the features associated to the RGO sheets, and thus meaningful conclusions could not be reached. To remove the biomolecules, the reduced dispersions were first destabilized by 
the addition of $37 \% \mathrm{HCl}$ solution until flocculation. The solid aggregate was subsequently transferred to filter paper and extensively washed with Milli-Q water for several days. Finally, the solid was re-suspended in a water:ethanol mixture (1:4 by volume) by bath sonication ( $5 \mathrm{~h}$ ) and the resulting suspension was vacuum-filtered to yield the free-standing RGO film.

Atomic force microscopy (AFM) measurements were performed under ambient conditions $\left(\sim 22-24{ }^{\circ} \mathrm{C}, \mathrm{RH} \sim 40 \%\right)$ with a Nanoscope IIIa Multimode apparatus (Veeco Intruments) in the tapping mode of operation. Rectangular Si cantilevers with spring constant of $\sim 40 \mathrm{~N} \mathrm{~m}^{-1}$ and resonance frequency $\sim 250-300 \mathrm{kHz}$ were used. Samples for AFM were prepared by drop-casting a small volume of diluted suspension ( 0.01-0.02 $\mathrm{mg} \mathrm{mL} \mathrm{m}^{-1}$ of $\left.\mathrm{RGO}\right)$ onto a pre-heated $\left(\sim 50-60^{\circ} \mathrm{C}\right)$ highly oriented pyrolitic graphite (HOPG) substrate, which was subsequently rinsed with Milli-Q water and allowed to dry. Scanning transmission electron microscopy (STEM) was performed on a Quanta FEG 650 system (FEI Company) operated at $30 \mathrm{kV}$. Transmission electron microscopy (TEM) images were recorded with a JEOL $2000 \mathrm{EX}-\mathrm{II}$ instrument operated at $160 \mathrm{kV}$. Specimens for both STEM and TEM were prepared by mixing equal volumes of aqueous sample suspension and ethanol. Then, $40 \mu \mathrm{L}$ of the resulting mixture were drop-cast onto a copper grid (200 square mesh) covered with a carbon film (Electron Microscopy Science) and allowed to dry. 


\section{Results and Discussion}

\subsection{Reduction of graphene oxide with biomolecules}

The biomolecules that were tested here for their ability to reduce graphene oxide were mostly hydrosoluble vitamins, organic acids and amino acids, and were principally chosen on the basis of their known antioxidant or reducing activity in certain biochemical reactions. Therefore, it was expected that at least some of them could also act as effective reductants for graphene oxide. Indeed, vitamin $\mathrm{C}$ has been previously shown to efficiently deoxygenate graphene oxide in aqueous and organic dispersion [22]. In the present work, other hydrosoluble vitamins, namely vitamin $\mathrm{B}_{6}$ (in two forms: pyridoxine and pyridoxamine dihydrochloride) [40,41] and vitamin $\mathrm{B}_{2}[(-)-$ riboflavin [42] and riboflavin 5'-monophosphate salt hydrate, the latter being a more soluble version of the former] were assayed. Also, the organic acids citric acid, fumaric acid, L-malic acid, and L-tartaric acid, all of which possess known antioxidant activity [43], were tested. Likewise, certain amino acids have been shown to reduce metallic ions [44] and have even been suggested to reduce graphene oxide (specifically, tyrosine) [36], but direct confirmation of the latter point has so far not been provided. Here, we selected a representative set of the 22 naturally occurring amino acids, as well as two peptides: L-arginine, L-asparagine, L-carnosine (dipeptide) [45], glycine, Lglutamic acid, L-gluthatione (tripeptide) [35,46], L-histidine, L-methionine [46], Lphenylalanine, L-tryptophan [46], and L-tyrosine [36,46]. In addition, $\alpha$-D-glucose [23], which together with L-gluthatione [35] has already been reported as a reductant for graphene oxide, was studied in an attempt to optimize its reducing efficiency.

To investigate and optimize the possible reducing effect of the biomolecules on the graphene oxide dispersions, two experimental variables were mainly taken into 
account: the biomolecule concentration in the dispersion and the reaction time (at 95 $\left.{ }^{\circ} \mathrm{C}\right)$. In general terms, for a given graphene oxide concentration and a given reductant, we can expect to have a concentration of reductant that is optimum for reducing the graphene oxide sheets. Above such concentration, further reactions between graphene oxide and reductant will not take place, whereas with lower concentrations removal of oxygen functional groups from graphene oxide will be limited to a fraction of those that can be actually eliminated with the optimum amount of that reductant [22]. Similarly, a certain time will be required for reaction completion, which can be expected to depend on the specific reductant used, as different reaction pathways with different kinetic barriers will probably come into play. With these considerations in mind, we set out to optimize the reducing effect of the selected biomolecules by probing different concentrations and reaction times.

Optimization of the reduction conditions was carried out for aqueous dispersions with a graphene oxide concentration of $0.1 \mathrm{mg} \mathrm{mL}^{-1}$. The extent of reduction achieved was initially followed with UV-vis absorption spectroscopy, by monitoring the position of the absorption maximum for the $\pi \rightarrow \pi^{*}$ transition of $\mathrm{C}=\mathrm{C}$ bonds $\left(\lambda_{\max }\right) . \lambda_{\max }$ is located at $\sim 231 \mathrm{~nm}$ for unreduced graphene oxide, but gradually red-shifts as deoxygenation proceeds and electronic conjugation $\left(\mathrm{sp}^{2}\right.$ character) is restored in the carbon lattice [5]. Furthermore, a good correlation between the extent of reduction and $\lambda_{\max }$, which reaches $\sim 268 \mathrm{~nm}$ for highly reduced samples, has been previously established in conjunction with other characterization techniques [22]. In the present work, reduction was considered to be successful when the value of $\lambda_{\max }$ for the graphene oxide dispersion after reaction with a given biomolecule was larger than 259$261 \mathrm{~nm}$. A value of $259-261 \mathrm{~nm}$ for $\lambda_{\max }$, which amounts to a relatively limited degree 
of reduction of the graphene oxide sheets, can be accomplished by simply heating the suspension at $95^{\circ} \mathrm{C}$ under basic conditions ( $\mathrm{pH}$ 10, achieved with, e.g., ammonia) in the absence of any reducing agent [22]. Because the experiments are carried out in basic medium ( $\mathrm{pH}$ 10) to promote the colloidal stability of the dispersions [5], a potential reducing agent can only be considered to be successful in that role if it affords values of $\lambda_{\max }$ clearly above $259-261 \mathrm{~nm}$.

\begin{tabular}{|c|c|c|c|c|}
\hline Reagent & $\begin{array}{l}\lambda_{\max } \\
(\mathrm{nm})\end{array}$ & $\begin{array}{c}\text { Reduces } \\
\text { graphene oxide? }\end{array}$ & $\begin{array}{c}\text { Concentration } \\
(\mathrm{mM})\end{array}$ & $\begin{array}{l}\text { Time } \\
\text { (h) }\end{array}$ \\
\hline Pyridoxine (PN) & 267 & Yes & 0.25 & 10 \\
\hline $\begin{array}{l}\text { Pyridoxamine dihydrochloride } \\
\text { (PM) }\end{array}$ & 267 & Yes & 0.25 & 8 \\
\hline (-)-riboflavin $\left(\mathrm{B}_{2}\right)$ & 268 & Yes & 2 & 8 \\
\hline $\begin{array}{l}\text { riboflavin 5'-monophosphate } \\
\text { sodium salt hydrate }\left(\mathrm{B}_{2} \text { salt }\right)\end{array}$ & 268 & Yes & 2 & 8 \\
\hline citric acid & 256 & No & 0.25 & 7 \\
\hline fumaric acid & 255 & No & 0.025 & 6 \\
\hline L-malic acid & 253 & No & 2.5 & 10 \\
\hline L-tartaric acid & 257 & No & 2 & 7 \\
\hline L-arginine (Arg) & 266 & Yes & 1 & 8 \\
\hline L-aspargine & 258 & No & 0.25 & 6 \\
\hline L-carnosine & 254 & No & 4 & 7 \\
\hline glycine & 259 & No & 10 & 6 \\
\hline L-glutamic acid & 257 & No & 0.5 & 6 \\
\hline L-glutathione (GSH) & 264 & Yes & 1 & 3 \\
\hline L-histidine (His) & 265 & Yes & 4 & 8 \\
\hline L-methionine & 261 & No & 2 & 4 \\
\hline L-phenylalanine & 257 & No & 1 & 6 \\
\hline L-tryptophan (Tryp) & 267 & Yes & 2 & 8 \\
\hline L-tyrosine & 261 & No & 2 & 8 \\
\hline$\alpha$-D-glucose $(\mathrm{Glu})$ & 267 & Yes & 0.25 & 3 \\
\hline
\end{tabular}

Table 2. Position of the absorption peak corresponding to $\pi \rightarrow \pi^{*}$ transitions of $\mathrm{C}=\mathrm{C}$ bonds $\left(\lambda_{\max }\right)$ for aqueous graphene oxide dispersions treated with different biomolecules. The biomolecule concentration and reaction time have been optimized to yield $\lambda_{\max }$ values as red-shifted as possible, indicating optimization of the reduction degree of graphene oxide. A given biomolecule is considered to be effective as a 
reductant when the value of $\lambda_{\max }$ it affords is well above that obtained just by heating the graphene oxide dispersion in basic medium (259-261 nm, ref. 22). The acronyms used throughout the text for the successful reductants are included.

Table 2 shows the values of $\lambda_{\max }$ attained with each of the 20 biomolecules under optimized conditions, together with the biomolecule concentration and reaction time required. On the basis of the above criterion, 9 out of the 20 tested reagents were found to be effective in reducing graphene oxide: pyridoxine (PN), pyridoxamine dihydrochloride (PM), riboflavin $\left(\mathrm{B}_{2}\right)$ and its phosphate salt $\left(\mathrm{B}_{2}\right.$ salt), arginine (Arg), gluthatione (GSH), histidine (His), tryptophan (Tryp), and glucose (Glu). Quite unexpectedly, some of the biomolecules (e.g., fumaric acid and L-malic acid) yielded $\lambda_{\max }$ values even lower than $259-261 \mathrm{~nm}$, suggesting not only that they are inefficacious as reducing agents for graphene oxide, but also that they hinder the partial reduction of the sheets that otherwise takes place under basic conditions [22]. As noticed from Table 2 , the effective biomolecules were seen to display somewhat different values of $\lambda_{\max }$ under optimized conditions, which indicates that they possess different reducing powers towards graphene oxide. According to such criterion, the weakest reducing agent would be GSH $\left(\lambda_{\max } \sim 264 \mathrm{~nm}\right)$, the strongest being $\mathrm{B}_{2}$ and $\mathrm{B}_{2}$ salt $\left(\lambda_{\max } \sim 268 \mathrm{~nm}\right)$. Under optimized conditions, Glu appeared to be also quite effective, exhibiting a $\lambda_{\max }$ value of $267 \mathrm{~nm}$, which has to be compared with a value of $261 \mathrm{~nm}$ reported beforehand for the same molecule under different conditions [23]. We note that, as explained above, a $\lambda_{\max }$ value of $259-261 \mathrm{~nm}$ is obtained in the absence of Glu just by heating the graphene oxide suspension at the same temperature and $\mathrm{pH}$ [22]. Likewise, a previous report ascribed the ability of the protein BSA to efficiently reduce graphene oxide to its 
tyrosine (Tyr) residues [36]. However, in the present work free Tyr molecules did not succeed in reducing the graphene oxide dispersions. As $\mathrm{pH}$ has been reported to be crucial in the performance of Tyr as a reducing agent in other cases, here the reaction was conducted both at the usual $\mathrm{pH}(\sim 10)$ (see Experimental Section) and at the $\mathrm{pH}$ reported to be optimum ( $\mathrm{pH} \sim 12)$ [47], but the same (negative) result was obtained in these two cases $\left(\lambda_{\max } \sim 261 \mathrm{~nm}\right)$. To explain such results, it can be argued that the reducing ability of Tyr residues in BSA towards graphene oxide could be greater than that of free Tyr molecules due to, e.g., synergistic effects with other residues. Alternatively, other residues present in BSA could be responsible for the reduction of graphene oxide instead of its Tyr residues. In this regard, a good candidate would be the Arg residues, given that such amino acid in free form was shown here to successfully reduce graphene oxide (see Table 2). We note that Arg residues outnumber their Tyr counterparts in the BSA molecule (42 vs. 21 , respectively).

Digital pictures of aqueous RGO dispersions prepared using the aforementioned nine successful reductants under optimized conditions are shown in Fig. 1, together with that of the starting, unreduced graphene oxide dispersion. The black and strongly opaque tone of the dispersions reacted with these biomolecules, as compared with the yellow-brown color characteristic of the starting dispersion, can be considered as a clear indication of efficient reduction. These reduced dispersions were seen to be stable for at least several months, except for samples reduced with Arg, which tended to aggregate within several days. 


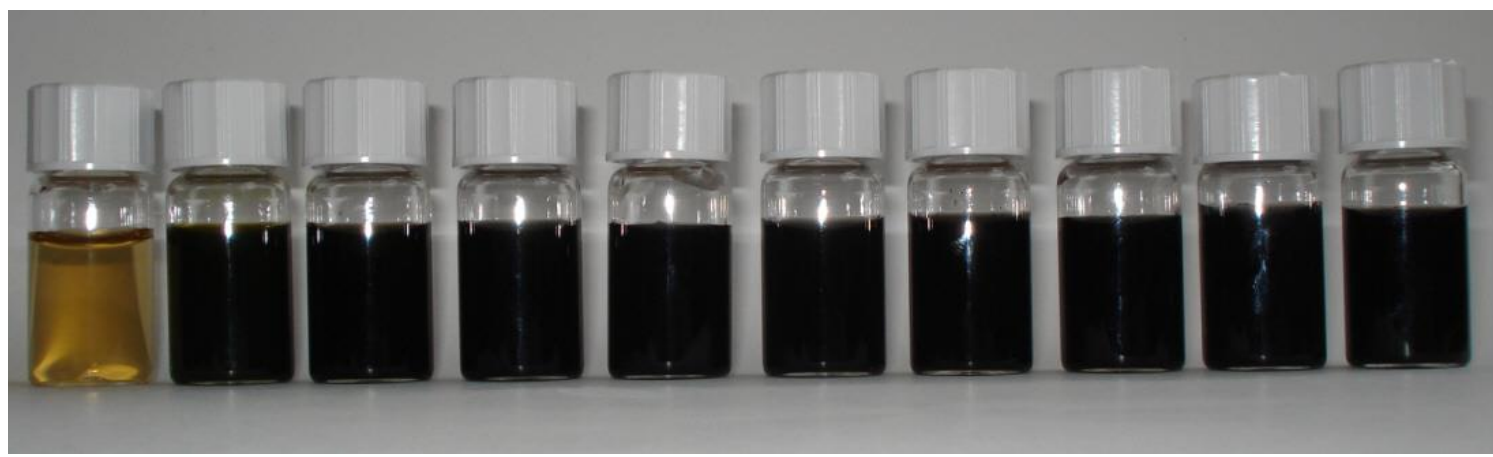

Figure 1. Digital picture of unreduced and biomolecule-reduced graphene oxide dispersions in water. From left to right: unreduced dispersion and dispersions reduced with $\mathrm{B}_{2}$ salt, $\mathrm{B}_{2}$, Glu, His, PN, PM, Arg, GSH and Tryp.

To confirm the successful reduction of the graphene oxide sheets, the dispersions were processed into free-standing paper-like films, as detailed in the Experimental Section, and characterized by TGA, ATR-FTIR spectroscopy, XPS and measurement of their sheet resistance. Fig. 2 shows thermogravimetric (TG, panel a) and differential thermogravimetric (DTG, panel b) plots of unreduced graphene oxide and graphene oxide reacted with the different effective bioreductants under the optimized conditions indicated in Table 2. The plots corresponding to the riboflavin salt ( $\mathrm{B}_{2}$ salt) are very similar to those of $\mathrm{B}_{2}$ and are not shown to avoid repetition. It can be noticed that unreduced graphene oxide exhibits a significant weight loss $(\sim 30 \%)$ in a narrow temperature range $\left(\sim 180-240{ }^{\circ} \mathrm{C}\right)$, which has been previously attributed to the decomposition of its labile oxygen functional groups [48]. Such a sharp weight loss step was no longer observed for graphene oxide reacted with the biomolecules (weight losses typically $\sim 5 \%$ ), indicating that most of these oxygen functional groups were eliminated. When using $\mathrm{B}_{2}$ as a reductant, a small weight loss step ( 5\%) appeared around $265^{\circ} \mathrm{C}$ (see Fig. 2a). This feature was never observed for unreduced graphene oxide or graphene oxide reduced with the other biomolecules, and can be tentatively 
attributed to remnants of riboflavin and/or its oxidized product that interact strongly with the RGO sheets and thus could not be fully washed away.

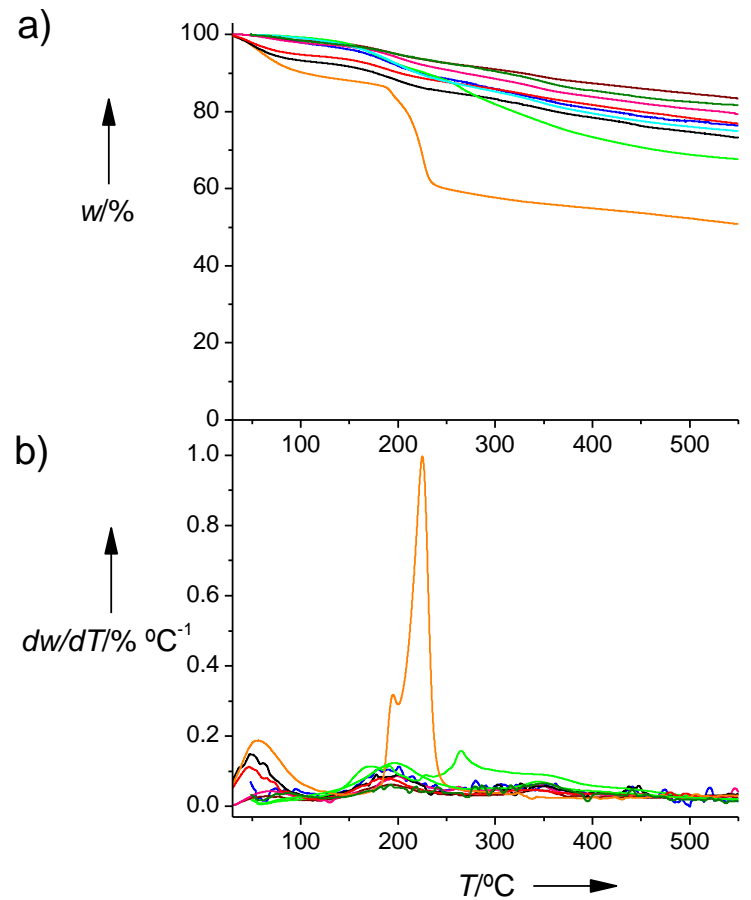

Figure 2. (a) Thermogravimetric (TG) and (b) differential thermogravimetric (DTG) plots recorded under Ar flow for paper-like films of unreduced graphene oxide (orange) and of graphene oxide reduced with PN (cyan), PM (red), $\mathrm{B}_{2}$ (fluorescent green), Arg (blue), GSH (pink), His (black), Tryp (olive green) and Glu (wine).

The significant decrease in the amount of oxygen functional groups in graphene oxide upon reaction with the bioreductants was further confirmed by the drop in the intensity of all the absorption bands associated to vibrations of such groups in the corresponding ATR-FTIR spectra (Fig. 3). For unreduced graphene oxide (Fig. 3, orange plot), bands at $3000-3500 \mathrm{~cm}^{-1}$ (OH stretching), $\sim 1720 \mathrm{~cm}^{-1}(\mathrm{C}=\mathrm{O}$ stretching), 
$1400 \mathrm{~cm}^{-1}$ (O-H bending vibrations), 1300-1350 $\mathrm{cm}^{-1}$ (C-OH stretching), $1220 \mathrm{~cm}^{-1}$ (breathing vibrations of epoxy groups) and $980 \mathrm{~cm}^{-1}$ (vibrations of epoxy, ether or peroxide groups) were clearly observed. By contrast, the intensity of all these bands diminished considerably for the samples treated with any the aforementioned successful bioreductants. The ATR-FTIR spectra of samples reduced with $\mathrm{B}_{2}$ and $\mathrm{B}_{2}$ salt (Fig. 3, bright green and yellow plots, respectively) support the idea that, despite the extensive washing step, some residual reducing agent was retained on the RGO sheets, as already suggested by the TGA data. Indeed, the intensity of the absorption bands related to hydroxyl groups, which are present in these two molecules in a significant proportion (see Table 1), is high in relation to that of graphene oxide reacted with any of the other 7 successful bioreductants.

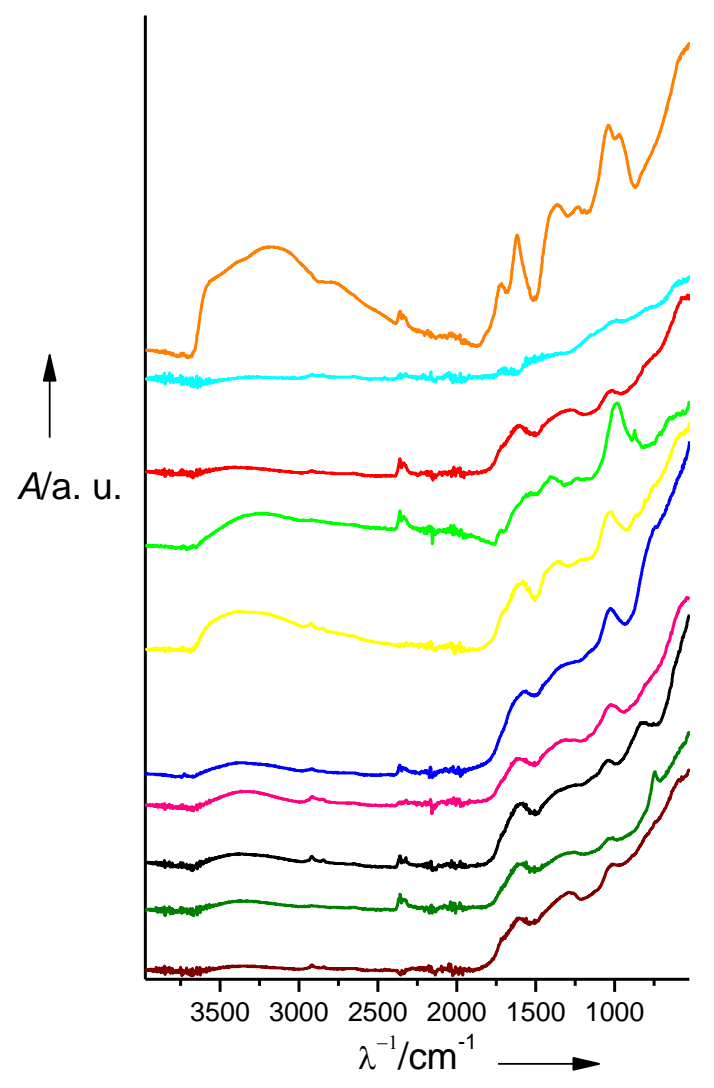


Figure 3. ATR-FTIR spectra of paper-like films of unreduced graphene oxide (orange) and graphene oxide reduced with PN (cyan), PM (red), $\mathrm{B}_{2}$ (fluorescent green), $\mathrm{B}_{2}$ salt (yellow), Arg (blue), GSH (pink), His (black), Tryp (olive green) and Glu (wine).

XPS survey spectra confirmed the effective deoxygenation of graphene oxide by the identified bioreductants: the $\mathrm{O} / \mathrm{C}$ atomic ratio measured on the different paper-like samples dropped from 0.43 in unreduced graphene oxide to $0.13-0.19$ after reaction with these biomolecules. Such $\mathrm{O} / \mathrm{C}$ ratios are similar to those obtained from similar reduction processes [39] although somewhat higher than that obtained when hydrazine is used under optimized conditions $(\mathrm{O} / \mathrm{C}$ ratio $\sim 0.10)$ [22]. It must be noted that deoxygenation and reduction, although sometimes used as synonyms, are not equivalent. Deoxygenation can occur without reduction, e. g., two vicinal hydroxyls to yield one epoxy group (and one water molecule). Reduction can occur without deoxygenation as well, e. g., one epoxy group transforming into one hydroxyl group. However, the oxidation state of the $\mathrm{C}$ atoms in RGO can be clearly ascertained from the high resolution C1s core level XPS spectra shown in Fig. 4, so that the actual reduction of graphene oxide by reaction with the efficient bioreductants can be confirmed. Unreduced graphene oxide (Fig 4, orange plot) exhibits two main components at 284.6 and $286.6 \mathrm{eV}$, which can be ascribed, respectively, to carbon atoms in an unoxidized, graphitic environment and to carbon atoms single-bonded to oxygen (e.g., from hydroxyl or epoxy groups) [39]. A third, minor component is located at about $287.9 \mathrm{eV}$ and can be attributed to carbon atoms double-bonded to oxygen (e.g., carbonyl groups). As clearly noticed from Fig 4, treatment of graphene oxide with any of the bioreductants prompted a significant decrease in the relative contribution of oxygen- 
bonded carbon to the XPS C1s signal (especially carbon single-bonded to oxygen). Concomitantly, a wide and weak (but nonetheless detectable) component was seen to develop at $\sim 291 \mathrm{eV}$ for the biomolecule-treated samples, which can be assigned to the $\pi \rightarrow \pi^{*}$ shake-up satellite peak typical of graphitic materials [49]. It is known that deoxygenation occurs to a certain extent just treating graphene oxide under mild basic medium and that a limited degree of reduction is attained in strong basic medium [50]. This last result is what we find if we just heat in $\mathrm{NH}_{3}$ at $95^{\circ} \mathrm{C}$ in the absence of any reductant, as can be seen in Fig 4 (navy plot). This matches with the already mentioned red-shift of $\lambda_{\max }$ to $259-261 \mathrm{~nm}$ in the UV-vis absorption spectrum for such dispersion. Such results clearly indicate that an effective reduction of graphene oxide by these biomolecules was achieved, and also that the electronically conjugated, graphitic character of the original carbon lattice could be restored to a substantial extent.

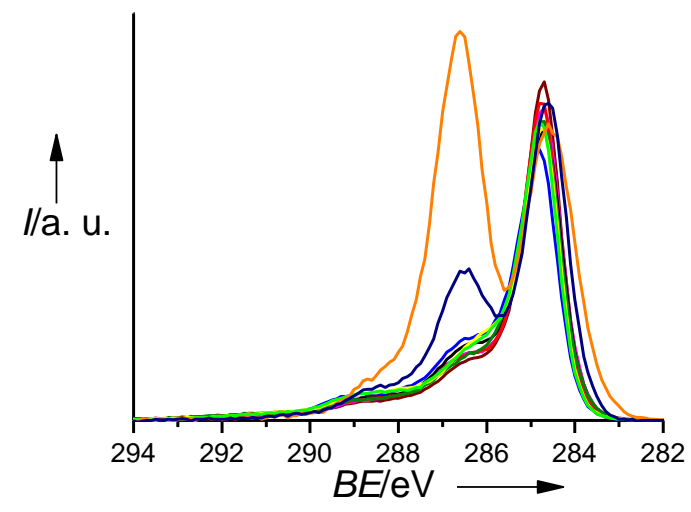

Figure 4. High resolution XPS C1s core level spectra recorded for unreduced graphene oxide (orange) and graphene oxide reduced with PM (red), $\mathrm{B}_{2}$ (fluorescent green), $\mathrm{B}_{2}$ salt (yellow), Arg (blue), GSH (pink), His (black), Tryp (olive green) and Glu (wine). Also the spectrum of a graphene oxide dispersion with $26 \mathrm{mM} \mathrm{NH}_{3}$ heated at $95{ }^{\circ} \mathrm{C}$ during $4 \mathrm{~h}$ has been added as a reference (navy blue). BE stands for binding energy. 
Another evidence of the restoration of the electronic conjugation is provided by measurement of the sheet resistance $\left(R_{s}\right)$ of the RGO films. The $R_{s}$ of graphene oxide before and after reduction with hydrazine has been reported to be in the order of $10^{10}$ $10^{11} \Omega /$ sq $[33,38]$ and $10^{5}-10 \Omega /$ sq [33,22], respectively. The RGO films obtained in this work through reduction in optimized conditions with different bioreductants yielded $\mathrm{R}_{\mathrm{s}}$ values of $8.99 \times 10^{2} \Omega / \mathrm{sq}(\mathrm{PN}), 7.57 \times 10^{2} \Omega / \mathrm{sq}(\mathrm{PM}), 1.70 \times 10^{6} \Omega / \mathrm{sq}\left(\mathrm{B}_{2}\right), 4.50 \times 10^{6}$ $\Omega / \mathrm{sq}\left(\mathrm{B}_{2}\right.$ salt), $4.52 \times 10^{4} \Omega / \mathrm{sq}$ (Arg), $1.39 \times 10^{3} \Omega / \mathrm{sq}(\mathrm{GSH}), 6.24 \times 10^{3} \Omega / \mathrm{sq}$ (His), $3.39 \times 10^{3} \Omega /$ sq (Tryp), and $2.77 \times 10^{2} \Omega /$ sq (Glu), respectively. The relatively low $\mathrm{R}_{\mathrm{S}}$ values, in the order of $10^{2}-10^{3} \Omega / \mathrm{sq}$, shown by some of the RGO films prepared in this study are lower than most of the $\mathrm{R}_{\mathrm{s}}$ values previously reported in the literature for RGO obtained with green reductants under mild conditions, indicating a high degree of chemical reduction (for a detailed comparison with previously reported values of sheet resistance and/or conductivity of RGO obtained by reduction with environmentallyfriendly reductants, see Table S1 in the Supplementary Material). In fact, amongst the previously reported green reductants, only vitamin $C$ yields lower $R_{s}$ values [22]. $B_{2}$ and $B_{2}$ salt show the highest $R_{s}$ values in this series of RGO films. This can be explained by the retention of some amount of reductant and/or its oxidized on the RGO samples despite extensive washing [24], as the TGA and ATR-FTIR spectroscopy data suggest (see also the X-ray diffraction data in the Supplementary Material). The fact that RGO dispersions obtained with $\mathrm{B}_{2}$ salt show a exceedingly good dispersion stability, better than that of any of the other dispersion, as will be explained below in detail, also points to strong adsorption of $\mathrm{B}_{2}$ salt and/or its oxidized form on the graphene sheets. The 
adsorption of $\mathrm{B}_{2}$ salt explains in turn that the corresponding paper-like films display the highest $\mathrm{R}_{\mathrm{s}}$ in the series.

Although aqueous dispersions of unreduced graphene oxide are made up of individual, single-layer sheets that are highly stable against aggregation and re-stacking, the same does not always hold true following removal of most of their oxygen functional groups in a reduction treatment [5]. This is mainly a consequence of the relatively low concentration of hydrophilic and/or ionizable oxygen-containing groups that remain in well-reduced graphene oxide samples. To investigate the dispersion state of the RGO sheets prepared with the present bioreductants, their aqueous suspensions were drop-cast onto atomically flat HOPG substrates and imaged by AFM (Fig. 5). The results indicated that the reduced sheets did not agglomerate and were kept as individual single-layer objects in their corresponding dispersions, even several months after the reduction treatment was carried out. Fig. 5 illustrates this point with some representative AFM images obtained from dispersions reduced with Arg (a), GSH (b) and PM (c). Similar to the case of the unreduced dispersions (images not shown), the reduced ones were seen to be constituted by irregular (polygonal) objects a few to several hundred $\mathrm{nm}$ in lateral size and $\sim 1 \mathrm{~nm}$ of apparent thickness (measured as their height relative to the HOPG substrate), which indicates that they indeed correspond to single-layer sheets [51]. The only notable exception to the long-term colloidal stability observed for the single-layer RGO sheets was, as commented above, that of dispersions reduced with Arg, which were only stable for a few days and tended to agglomerate afterwards. On the other hand, dispersions reduced with $\mathrm{B}_{2}$ salt were seen to be exceedingly stable compared to those prepared with the other bioreductants, even under conditions that are usually considered extremely unfavorable to their stability (e.g., at low pH) [5]. For 
example, addition of small amounts of concentrated $\mathrm{HCl}$ solution readily destabilized all the biomolecule-reduced dispersions, except those prepared with $\mathrm{B}_{2}$ salt, which required much larger volumes of the acid to achieve destabilization. The origin of this behavior can be mainly traced to the following two facts: (i) as mentioned before, $\mathrm{B}_{2}$ salt molecules (or their oxidized product) are strongly adsorbed onto the RGO sheets, possibly by way of $\pi-\pi$ interactions between their aromatic domains, and (ii) adsorbed $\mathrm{B}_{2}$ salt molecules provide colloidal stability to the RGO sheets through electrostatic repulsion afforded by the negatively charged organophosphate group of the salt (see Table 1), even under significantly acidic conditions (the first $\mathrm{pK}_{\mathrm{a}}$ of phosphoric acid is 2) [52]. Thus, in addition to being an effective reductant of graphene oxide, $B_{2}$ salt (or its oxidized product) can also be used as a powerful stabilizer of the reduced
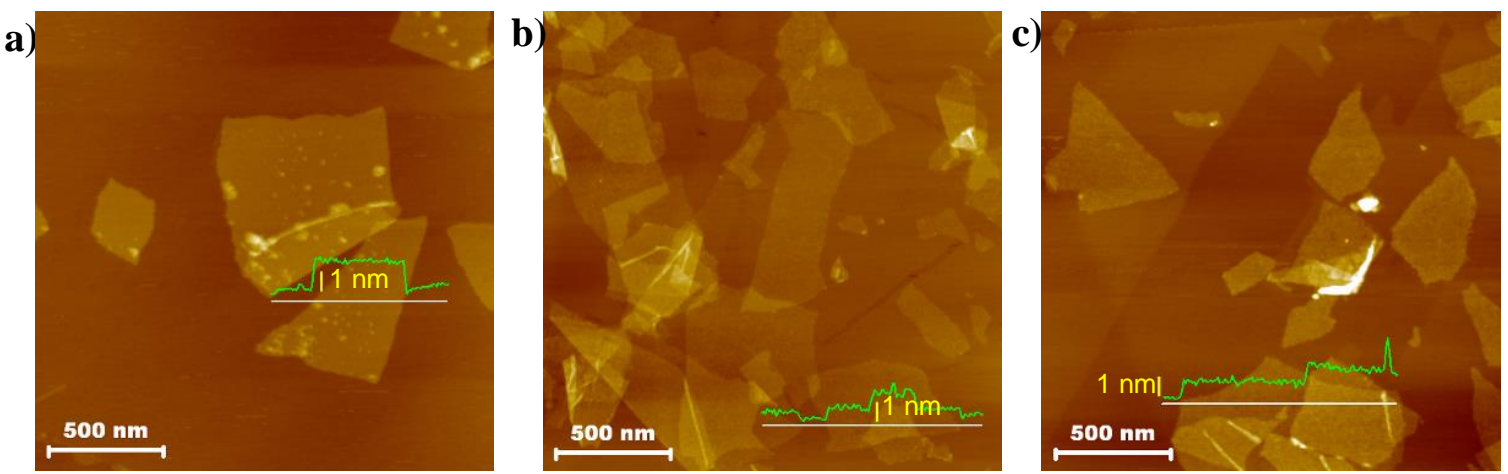
dispersions, which can be advantageous with a view to their further processing.

Figure 5. AFM images of graphene oxide sheets reduced with Arg (a), GSH (b) and PM (c) deposited onto HOPG substrates from their corresponding aqueous dispersions. In each case, a representative line profile (green line) taken along the marked white line is shown overlaid on the image. 
The mechanism for the removal of oxygen functional groups from graphene oxide by reaction with reducing agents is still an open question even in the case of those most widely employed to this end (e.g., hydrazine). Although some reduction mechanisms involving $-\mathrm{OH}$ and/or $-\mathrm{NH}_{2}$ groups from the reductant have been put forward $[21,23,27,33,35]$, they are generally speculative and mainly based on previous knowledge of the reactions between a given reductant and small organic molecules, but not graphene oxide. A first step in the experimental determination of reduction pathways is the identification of the oxidation products of the reducing agent after reaction with graphene oxide, but in most cases such work has not been carried out $[21,23,33,35]$. The identification of oxidation products for the newly reported bioreductants ( $\mathrm{PN}, \mathrm{PM}, \mathrm{B}_{2}$, etc) by high performance liquid chromatography/mass spectrometry is currently being undertaken by us, and the results will be documented elsewhere. Preliminary results indicate that, for example, reaction of both PN and PM with graphene oxide yields pyridoxal, which in turn suggests that oxidation of these two bioreductants proceeds through transformation of their hydroxyl and amine groups, respectively, into an aldehyde (see the Supplementary Material for additional data and suggested reaction mechanisms for the rest of the bioreductants). We also note from Table 2 that none of the tested organic acids succeeded in deoxygenating graphene oxide under the basic conditions that were employed here with all the potential reducing agents, implying that these molecules possess a poor reducing ability. However, it should be stressed that some strong inorganic acids, such as HI, have proved to be particularly effective reductants of graphene oxide [53,54]. Liao et al have suggested that a highly acidic aqueous environment is able to catalyze dehydration reactions of hydroxyl and epoxy groups in graphene oxide, hence leading to its reduction [55]. To 
test whether the present organic acids are also able to induce the reduction of graphene oxide by the generation of a sufficiently acidic environment, we heated as-prepared, non-basified graphene oxide dispersions in the presence of the organic acids at $95{ }^{\circ} \mathrm{C}$. In these cases, the $\mathrm{pH}$ of the medium was around 3, but no signs of reduction were observed with any of the acids even after long heating times. In the light of this, we hypothesize that the organic acids investigated here are too weak compared with, e.g., HI $\left(\mathrm{pK}_{\mathrm{a}}\right.$ values of $\sim 3$ (organic acids) vs. $\sim-10(\mathrm{HI})$ ) [52] to induce significant dehydration reactions, and therefore reduction, in graphene oxide.

\subsection{Liquid-phase synthesis and catalytic activity of RGO-supported silver nanoparticles}

The synthesis of metal nanoparticles is an active area of research due to their potential utility in, e.g., catalysis, photonics, biological labelling or sensing [56]. A simple, easily scalable method towards the preparation of metal nanoparticles in colloidal dispersion is based on the reduction of a metal salt precursor, usually in the presence of a stabilizer [44]. The use of carefully selected reductants and/or stabilizers is the cornerstone of this synthetic approach, given that their specific nature generally impacts the characteristics and performance of the resulting nanoparticles [44,57] and also that there is a growing need to develop more sustainable preparation processes [58]. In this context, the identification of effective bioreductants for graphene oxide, as reported in the previous section, can be exploited for the preparation of RGO-metal nanoparticle hybrids by simultaneous reduction of graphene oxide and a metal salt. The prospective application of these hybrids in different technologically relevant areas has made them the focus of significant current interest $[59,60]$. More specifically, Ag NPs are good candidates for use in catalysis, as silver is relatively cheap and a very active catalyst in several reactions [57,61]. Many of the effective bioreductants investigated in the previous 
section, including Glu, Arg, $\mathrm{B}_{2}$, Tryp and His have already been employed for the preparation of unsupported Ag NPs in aqueous medium [62-66]. However, to the best of our knowledge, there are no previous reports on the use of either PN or PM for the synthesis of metal nanoparticles (either supported or unsupported).

In the present work, RGO-Ag NP hybrids were prepared by the simultaneous reduction of graphene oxide and $\mathrm{Ag}(\mathrm{I})$ (in the form of $\mathrm{AgNO}_{3}$ ) with the nine effective bioreductants of Table 2, without the need to use any additional reducing or stabilizing agents. Control experiments indicated that there was an optimum $\mathrm{AgNO}_{3}$ concentration (0.34 mM for $0.1 \mathrm{mg} \mathrm{mL}^{-1}$ graphene oxide dispersions) for the generation of $\mathrm{Ag} \mathrm{NPs}$ selectively onto the RGO sheets, as opposed to their generation in the bulk of the solution in the form of stand-alone, unsupported nanoparticles. Concentrations significantly below the optimum value did not bring about substantial numbers of $\mathrm{Ag}$ NPs, whereas higher concentrations tended to favor the growth of unsupported nanoparticles. Thus, subsequent experiments were accomplished by varying the concentration of bioreductant at the optimum $\mathrm{AgNO}_{3}$ concentration.

Some representative results of the spectroscopic (UV-vis absorption and XPS) and microscopic (AFM, STEM and TEM) characterization of the obtained hybrids are shown, respectively, in Figs. 6 and 7, and Table 3 summarizes their main features (particle size and density). The grayish tinge typical of diluted aqueous dispersions containing only RGO sheets (inset to Fig. 6a, right cuvette) was replaced by a yellowish tone when graphene oxide and $\mathrm{AgNO}_{3}$ were co-reduced with $4 \mathrm{mM}$ His (inset to Fig. 6a, left cuvette). Furthermore, UV-vis absorption spectra revealed the appearance of a feature at $\sim 420 \mathrm{~nm}$ for the latter (Fig. 6a), which can be ascribed to the well-known surface plasmon resonance (SPR) band characteristic of metallic silver nanostructures 
[67]. The generation of metallic silver in the dispersions was further confirmed by XPS measurements (Fig. 6b). Two well-defined and symmetrical peaks centered at 368.4 and $374.4 \mathrm{eV}$ were observed and assigned to photoelectrons ejected, respectively, from $3 \mathrm{~d}_{5 / 2}$ and $3 \mathrm{~d}_{3 / 2}$ levels of $\mathrm{Ag}^{0}$ [68]. No components at $\sim 367$ and $373 \mathrm{eV}$ were detected, indicating that oxidized silver $[\mathrm{Ag}(\mathrm{I})]$ was not present in the prepared materials in significant amounts.

a)

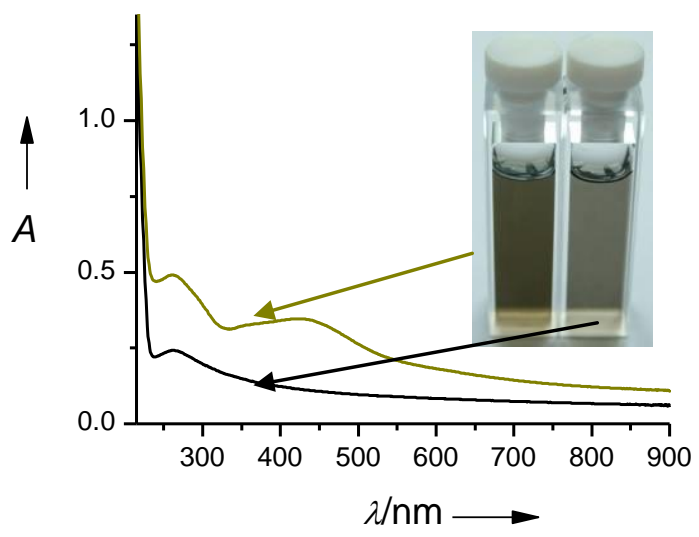

b)

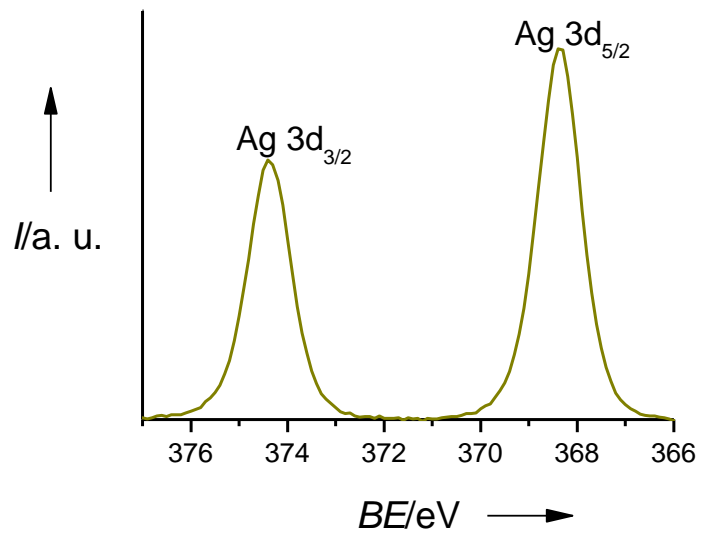

Figure 6. Spectroscopic characterization of RGO-Ag NP hybrids: (a) UV-vis absorption spectra and digital picture (inset) of a graphene oxide dispersion reduced with His in the absence (black curve and inset right) and presence (green curve and inset left) of $\mathrm{AgNO}_{3}$. (b) High resolution $\mathrm{Ag} 3 \mathrm{~d}$ core level spectrum recorded by XPS of a typical RGO-Ag NP hybrid. BE stands for binding energy.

Fig. 7 shows AFM (a,b), STEM (c,d) and TEM (e,f) images of RGO-Ag NP hybrids prepared with $2 \mathrm{mM}$ His (a), $1 \mathrm{mM}$ PM (b), $1 \mathrm{mM}$ Arg (c), $2 \mathrm{mM}$ Tryp (d), 2 $\mathrm{mM} \mathrm{B}_{2}$ salt (e) and $4 \mathrm{mM}$ His (f) as reductants. In all cases, the RGO sheets were found 
to be decorated with either white (AFM images) or black (bright-field STEM and TEM images) dots, which were never observed for graphene oxide sheets reduced in the absence of $\mathrm{AgNO}_{3}$ (see, e.g., Fig. 5 for the case of AFM), and were therefore attributed to the Ag NPs. As mentioned above, the preparation was performed under conditions (mainly determined by $\mathrm{AgNO}_{3}$ concentration) that led to Ag NPs exclusively associated to the sheets, without stand-alone nanoparticles being generated, and this is reflected in the images of Fig. 7 for the different hybrids. From the AFM images, the density of nanoparticles on the RGO sheets was estimated to be typically between $1-10 \mu \mathrm{m}^{-2}$, and higher resolution images by TEM (e.g., Fig. 7e and f) showed that they exhibit a faceted morphology. Estimation of nanoparticle size in the hybrids by TEM revealed it to be highly dependent on the bioreductant used and/or its concentration. This is apparent from the data collected in Table 3 for several selected hybrids. With a proper choice of bioreductant, nanoparticle sizes could be tuned in a range from 5-10 $\mathrm{nm}(0.1 \mathrm{mM} \mathrm{GSH})$ to $100-150 \mathrm{~nm}$ (3 $\mathrm{mM}$ Tryp). Only individual nanoparticles (with some occasional agglomerate) were observed in every hybrid except for that prepared with $4 \mathrm{mM} \mathrm{B}_{2}$, which consisted solely of agglomerates. The results of this local, TEM-based characterization of the nanoparticles were generally consistent with those obtained for the bulk of the hybrid in aqueous suspension by UV-vis absorption spectroscopy. It has been demonstrated that the SPR band of Ag NPs becomes progressively broader, more asymmetrical and red-shifted upon increasing their size from several nanometers to many tens of nanometers [67]. As a matter of fact, we observed such correlation between the UV-vis spectral features and the nanoparticle sizes estimated by TEM. We also note that the present bioreductants could be similarly used to prepare RGO hybrids with nanoparticles of other noble metals, for example gold using $\mathrm{HAuCl}_{4}$ as a precursor. 
A brief characterization of these hybrids is presented in the Electronic Supplementary Information.
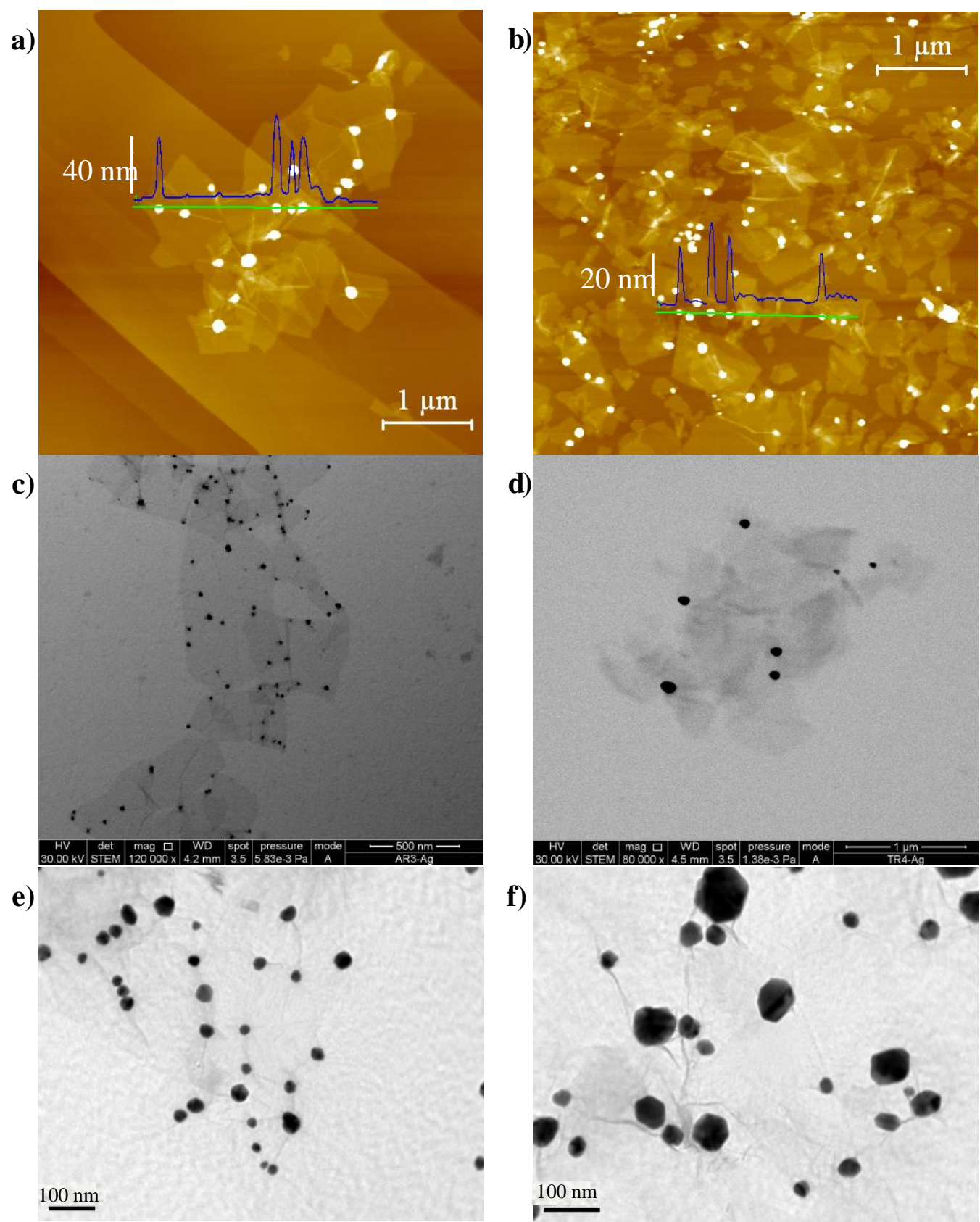

Figure 7. Microscopic characterization of RGO-Ag NP hybrids: (a,b) AFM images of hybrids prepared with $2 \mathrm{mM}$ His (a) and $1 \mathrm{mM}$ PM (b) deposited onto HOPG substrates. A representative line profile (blue line) taken along the marked green line is shown superimposed on each AFM image. (c,d) STEM images of hybrids prepared with $1 \mathrm{mM}$ 
Arg (c) and 2 mM Tryp (d). (e,f) Detailed TEM images of Ag NPs on RGO sheets obtained with $2 \mathrm{mM} \mathrm{B}_{2}$ salt (e) and $4 \mathrm{mM}$ His (f).

\begin{tabular}{lll}
\hline Reductant & $\begin{array}{l}\text { Concentration } \\
(\mathrm{mM})\end{array}$ & $\begin{array}{l}\text { Particle size } \\
(\mathrm{nm})\end{array}$ \\
\hline pyridoxine & 0.5 & $\sim 50-90$ \\
& 1 & $\sim 70-100$ \\
\hline pyridoxamine dihydrochloride & 1 & $\sim 20-25$ \\
& 1.5 & $\sim 40$ \\
\hline (-)-riboflavin & 2 & $\sim 20$ \\
& 4 & $-($ Only aggregates $)$ \\
\hline riboflavin salt & 2 & $\sim 25-50$ \\
& 4 & $\sim 25-50$ \\
\hline L-arginine & 1 & $\sim 20-30$ \\
& 2 & $\sim 20-40$ \\
\hline L-glutathione & 0.1 & $\sim 5-10$ \\
& 0.5 & $>100$ \\
& 1 & Only extended Ag structures formed \\
\hline L-histidine & 2 & $\sim 20-30$ \& 50-80 \\
& & (Bimodal distribution of NP diameter. The \\
& & smaller NP are more abundant). \\
& 6 & $\sim 50-80$ \\
\hline L-tryptophan & 2 & $\sim 50-150$ \\
& 3 & $\sim 100-150$ \\
\hline$\alpha$-D-glucose & 0.125 & $\sim 60-90$ \\
& 0.5 & $\sim 80$ \\
\hline
\end{tabular}

Table 3. Preparation conditions and microscopic features of RGO-Ag NP hybrids prepared with different bioreductants.

To evaluate the catalytic activity of the RGO-Ag NP hybrids, the reduction of $p$ nitrophenol to $p$-aminophenol with $\mathrm{NaBH}_{4}$ in aqueous medium at RT was investigated. Such a reaction constitutes a convenient model system to study the catalytic performance of metal nanoparticles and is also relevant from a practical perspective, because it is one of the main steps in the synthesis of widely employed pharmaceutical compounds (e.g., paracetamol) [69]. A selection of the hybrids prepared here was chosen for the catalytic experiments, namely, hybrids prepared with $2 \mathrm{mM}$ His, $1 \mathrm{mM}$ Arg and 1mM PM; the latter was chosen mainly because it had never been previously 
used in the synthesis of Ag NPs. The transformation of $p$-nitrophenol to $p$-aminophenol was followed by UV-vis absorption spectroscopy. Fig. 8a shows UV-vis spectra of aqueous solutions of (i) $p$-nitrophenol at the very slightly acidic $\mathrm{pH}$ of deionized water (black plot), (ii) deprotonated $p$-nitrophenol ( $p$-nitrophenoxide ion) that forms in the basic medium generated by the presence of $\mathrm{NaBH}_{4}$ (dark yellow plot), and (iii) the product of its reduction, $p$-aminophenoxide (violet plot). The strong absorption peak at $400 \mathrm{~nm}$ characteristic of $p$-nitrophenoxide is not present in its reduced counterpart, and thus such a peak can be used to monitor the reaction progress, i.e. the evolution of $p$ nitrophenoxide concentration with time. This is exemplified in Fig. $8 \mathrm{~b}$ for reaction of $p$ nitrophenoxide with $\mathrm{NaBH}_{4}$ in the presence of either the RGO-Ag NP hybrid prepared with PM (black squares) or stand-alone Ag NPs obtained by the standard and widely employed method of $\mathrm{NaBH}_{4}$ reduction of $\mathrm{AgNO}_{3}$ and stabilization with citrate anion (green squares) [67]. In these two cases, the experiments were performed with nanoparticles of similar size $(\sim 20-25 \mathrm{~nm})$ and same concentration $\left(\sim 3-4 \times 10^{9} \mathrm{~mL}^{-1}\right)$. An initial delay time ( 270-280 s) was observed, which can be ascribed to dissolved oxygen in the solution being consumed by $\mathrm{NaBH}_{4}$ [70]. Afterwards, reduction of $p$ nitrophenoxide proper came about and its absorbance at $400 \mathrm{~nm}$ was seen to decay in an exponential fashion. Considering also that an excess of $\mathrm{NaBH}_{4}$ was used in the experiments, the reduction can be regarded to proceed at a constant concentration of this reagent, and therefore should follow a pseudo-first-order kinetic behavior, so that,

$\frac{d[p-\mathrm{NP}]}{d \mathrm{t}}=-k_{a p p}[p-\mathrm{NP}]$

where $[p-\mathrm{NP}]$ is the concentration of $p$-nitrophenoxide and $k_{a p p}$ is the apparent rate constant of the reaction. Indeed, fitting of the experimental data of Fig. $8 \mathrm{~b}$ to an 
exponential function yielded residuals in the range of \pm 0.03 , confirming that Eq. (1) applies in the present case.
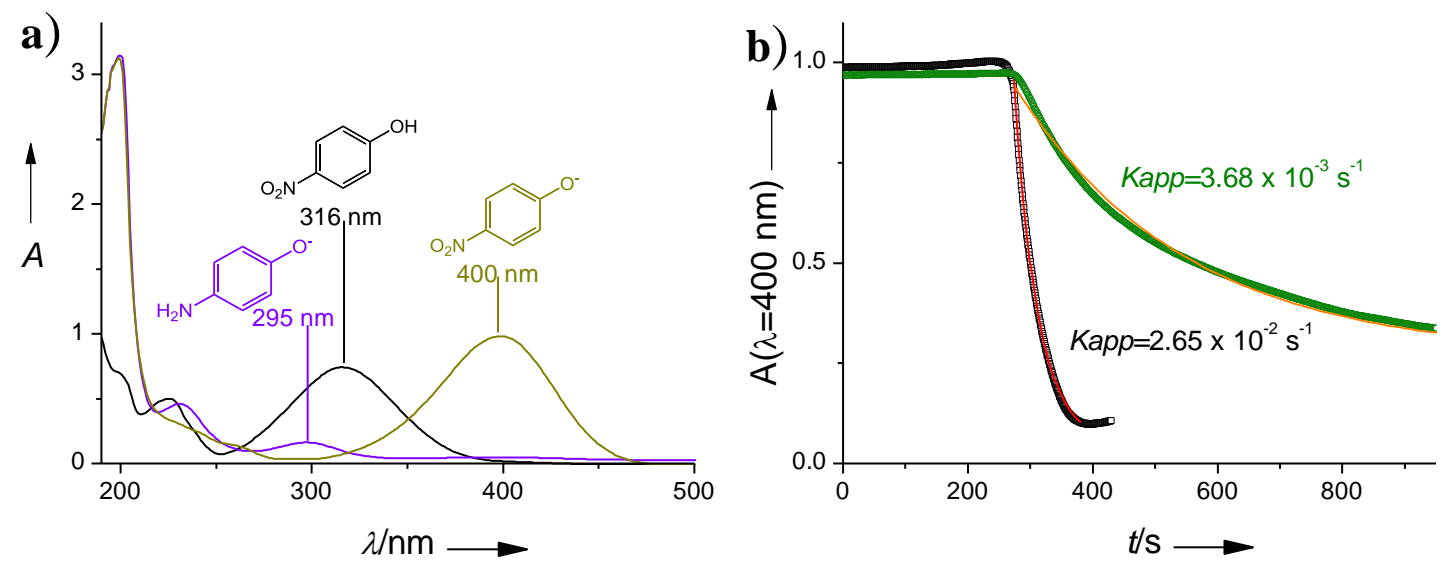

Figure 8. (a) UV-vis absorption spectra of $p$-nitrophenol (black curve), pnitrophenoxide ion (dark yellow), and $p$-aminophenoxide ion (violet). The absorption peak at $400 \mathrm{~nm}$ of $p$-nitrophenoxide is used to monitor its conversion to $p$ aminophenoxide by reduction with $\mathrm{NaBH}_{4}$. (b) Plot of absorbance at $400 \mathrm{~nm}$ for the reduction of $p$-nitrophenoxide with $\mathrm{NaBH}_{4}$ catalysed with RGO-Ag NP hybrid prepared with PM (black squares) and with stand-alone, citrate-capped Ag NPs prepared by $\mathrm{NaBH}_{4}$ reduction of $\mathrm{AgNO}_{3}$ following standard procedures (green squares). The experimental kinetic profiles could be well fitted to exponential decay functions, which are shown as overlaid red and orange lines, respectively. Experimental conditions: [ $p$ nitrophenol $]=0.06 \mathrm{mM} ;\left[\mathrm{NaBH}_{4}\right]=0.018 \mathrm{M} ;[\mathrm{Ag} \mathrm{NP}] \sim 0.2 \mu \mathrm{g} \mathrm{mL}^{-1}$.

The catalytic experiments yielded $k_{a p p}$ values of $5.37 \times 10^{-2}, 2.65 \times 10^{-2}$ and $2.64 \times 10^{-2} \mathrm{~s}^{-1}$ for RGO-Ag NP hybrids prepared with Arg, PM and His, respectively, 
whereas a value of $3.68 \times 10^{-3} \mathrm{~s}^{-1}$ was obtained with stand-alone, citrate-capped Ag NP synthesized by standard procedures. In general, the catalysts were seen to be quite stable in the short-term, as $k_{a p p}$ did not change significantly over 10 consecutive reaction cycles. Furthermore, to allow comparison of these catalytic activities on an equal footing, and also to compare them with data from the literature, the apparent rate constants were normalized to the surface area of silver employed per unit volume of dispersion (areal rate constant), and the results are $7.61,4.63,0.56 \mathrm{~L} \mathrm{~m}^{-2} \mathrm{~s}^{-1}$ for RGOAg NP hybrids prepared with Arg, PM and His, respectively, whereas a value of $3.68 \times 10^{-3} \mathrm{~s}^{-1}$ was obtained with stand-alone, citrate-capped Ag NPs (for a comparison with previously reported values measured for the same reaction using other Ag NPbased catalysts, see Table S2). To the best of our knowledge, the present Arg- and PMderived RGO-Ag NP hybrids exhibit the highest catalytic activities that have ever been documented for Ag NPs in this reaction (areal rate constants 3-5 times higher than the previous largest values [70-78].). By contrast, the performance of the His-derived hybrids is comparable (slightly lower) to that of the standard, citrate-capped Ag NPs. So far, the largest value of areal rate constant $\left(1.41 \mathrm{~L} \mathrm{~m}^{-2} \mathrm{~s}^{-1}\right)$ had been very recently achieved for a system made up of stand-alone nanoparticles synthesized by $\mathrm{NaBH}_{4}$ reduction of $\mathrm{AgNO}_{3}$ and stabilized by dextran [71].

We also note that the areal rate constants of the hybrids were obtained with justprepared hybrids. Nevertheless, another relevant issue that should be taken into account when assessing the performance of these catalysts concerns their colloidal stability in the medium where the catalytic reaction is carried out (water in the present case). Agglomeration of the metal nanoparticles in the reaction medium inevitably leads to the degradation of their catalytic performance, however active they are as individual 
entities, thus ruining their actual utility in the long-term. For this reason, the nanoparticles are frequently stabilized by, e.g., anchoring them onto suitable substrates or by coating them with different types of polymers or stabilizers. Although the latter option may limit the catalytic activity of the metal nanoparticles as a result of diffusional barriers between the reactants in the aqueous medium and the metal surface, such a disadvantage is usually far surpassed by the benefit of having a colloidally very stable catalyst that can be used for a very long time. In our case, even though the Argderived hybrid displayed a very high catalytic activity, the hybrid was not colloidally stable and precipitated in a matter of just a few days, similar to the stand-alone RGO sheets prepared with the same bioreductant, as noted beforehand. By contrast, the RGOAg NP hybrid prepared with PM boasted a combination of very high catalytic activity (4.63 $\mathrm{L} \mathrm{m}^{-2} \mathrm{~s}^{-1}$ ) and long-term colloidal stability. For example, aqueous dispersions of the PM-derived hybrids did not show any visible sign of agglomeration or precipitation and their catalytic activity was not significantly degraded nine months after their preparation. Therefore, the PM-derived hybrids constitute an exceptional catalyst for the studied reaction.

Finally, we briefly discuss the possible origins of the high catalytic activity of these hybrids. In principle, such activity could be either an intrinsic feature of the Ag NPs or a substrate (RGO)-promoted effect, or a combination of both. For example, relatively strong $\pi-\pi$ interactions between $p$-nitrophenol molecules and the RGO sheets could contribute to an increased concentration of this reactant in the neighborhood of the Ag NPs, thus increasing the reaction rate. To investigate this point, we synthesized Ag NPs using PM as a reductant and following the same procedure as described above, but in the absence of graphene oxide sheets. This resulted in stand-alone nanoparticles 
with somewhat larger diameters than those of their RGO-supported counterpart but similar areal rate constants, implying that their remarkably high catalytic activity is mainly an intrinsic feature of the Ag NPs and is not caused by the RGO substrate or by substrate-nanoparticle interaction effects. Still, using the RGO sheets as a support for the Ag NPs is advantageous in that the resulting hybrid displayed long-term colloidal stability in water, whereas the stand-alone PM-derived Ag NPs were less stable and tended to agglomerate. Although the origin of the high activity of these nanoparticles remains unknown, one plausible hypothesis could be that these bioreductants, or their oxidation products, tend to favor the growth and stabilization of Ag NPs exhibiting larger fractions of reactive sites and/or crystal planes on their surface, to the detriment of less reactive ones, thus leading to a higher catalytic activity [79].

\section{Conclusions}

A comprehensive survey using 20 biomolecules, selected on the basis of their known antioxidant and/or reducing ability in the biochemical context, has afforded the identification of several new efficient reductants for graphene oxide. The successful bioreductants are vitamins and amino acids, and include pyridoxine and pyridoxamine (vitamin $\mathrm{B}_{6}$ ), riboflavin (vitamin $\mathrm{B}_{2}$ ), arginine, histidine and tryptophan. These findings significantly increase the pool of innocuous and safe biomolecules available at present for reduction of graphene oxide and could thus facilitate the implementation of green approaches towards the production of graphene-based materials with improved performance for certain applications, where the selection of appropriate bioreductants could be relevant. As an example of this possibility, reduced graphene oxide-silver nanoparticle hybrids were prepared in aqueous medium using the newly identified bioreductants. In particular, hybrids made with pyridoxamine were shown to exhibit a 
combination of long-term colloidal stability and an unprecedented level of catalytic activity among silver nanoparticle-based catalysts in the reduction of $p$-nitrophenol with $\mathrm{NaBH}_{4}$, making them an excellent candidate for such application. The exploration of further benefits of using selected bioreductants to the performance of graphene-based materials is currently under way.

\section{Acknowledgements}

Financial support from the Spanish MINECO and the European Regional Development Fund (project MAT2011-26399) is gratefully acknowledged. M.J.F.-M. is thankful for the receipt of a pre-doctoral contract (FPI) from MINECO.

Supporting Information. Additional characterization of the RGO films by UV-vis spectroscopy, Raman spectroscopy and X-ray diffraction; comparison with previously reported RGO obtained with green reductants; proposed mechanisms for the bioreductants; comparison of areal rate constants obtained for the reduction of $\mathrm{p}$ nitrophenol with $\mathrm{NaBH}_{4}$ using a different $\mathrm{Ag}$ NP-based catalysts; general characterization of RGO-Au NP hybrids by UV-vis absorption spectroscopy and STEM.

\section{References}

[1] Luo B, Liu S, Zhi L. Chemical approaches toward graphene-based nanomaterials and their applications in energy-related areas. Small 2011; 8: 630-46.

[2] Yuxin L, Xiaochen D, Peng C. Biological and chemical sensors based on graphene materials. Chem Soc Rev 2012; 41: 2283-307. 
[3] Machado BF, Serp P. Graphene-based materials for catalysis. Catal Sci Technol 2012; $2:$ 54-75.

[4] Stankovich S, Dikin DA, Piner RD, Kohlhaas KA, Kleinhammes A, Jia Y, et al. Synthesis of graphene-based nanosheets via chemical reduction of exfoliated graphite oxide. Carbon 2007; 45: 1558-65.

[5] Li D, Müller MB, Gilje S, Kaner RB, Wallace GG. Processable aqueous dispersions of graphene nanosheets. Nature Nanotech 2008; 3: 101-5.

[6] Park S, Ruoff RS. Chemical methods for the production of graphenes. Nature Nanotech 2009; 4: 217-24.

[7] Shin H-J, Kim KK, Benayad A, Yoon S-M, Park HK, Jung I-S, et al. Efficient reduction of graphite oxide by sodium borohydride and its effect on electrical conductance. Adv Funct Mater 2009; 19: 1987-92.

[8] Pei S, Cheng H-M. The reduction of graphene oxide. Carbon 2012; 50: 3210-28.

[9] Mao S, Pu H, Chen J. Graphene oxide and its reduction: modeling and experimental progress. RSC Advances 2012; 2: 2643-62.

[10] Ambrosi A, Chua CK, Bonanni A, Pumera M. Lithium aluminum hydride as reducing agent for chemically reduced graphene oxides. Chem Mater 2012; 24: 2292-8. [11] Xu C, Wang X, Zhu J. Graphene-Metal Particle Nanocomposites. J Phys Chem C 2008; 112: 19841-5.

[12] Williams G, Seger B, Kamat PV. $\mathrm{TiO}_{2}-\mathrm{Graphene}$ Nanocomposites. UV-assisted photocatalytic reduction of graphene oxide. ACS Nano 2008; 2: 1487-91.

[13] Akhavan O, Ghaderi E. Photocatalytic reduction of graphene oxide nanosheets on $\mathrm{TiO}_{2}$ thin film for photoinactivation of bacteria in solar light irradiation. J Phys Chem C 2009; 113: 20214-20. 
[14] Akhavan O. Graphene nanomesh by ZnO nanorod photocatalysts. ACS Nano 2010; 4: 4174-80.

[15] Ng YH, Iwase A, Bell NJ, Kudo A, Amal R. Semiconductor/reduced graphene oxide nanocomposites derived from photocatalytic reactions. Catal. Today 2011; 164 : $353-7$.

[16] Akhavan O. Photocatalytic reduction of graphene oxides hybridized by $\mathrm{ZnO}$ nanoparticles in ethanol. Carbon 2011; 49: 11-8.

[17] Akhavan O, Choobtashani M, Ghaderi E. Protein degradation and RNA efflux of viruses photocatalyzed by graphene-tungsten oxide composite under visible light irradiation. J Phys Chem C 2012; 116: 9653-59.

[18] Paredes JI, Villar-Rodil S, Fernández-Merino MJ, Guardia L, Martínez-Alonso A, Tascón JMD. Environmentally friendly approaches toward the mass production of processable graphene from graphite oxide. J Mater Chem 2011; 21: 298-306.

[19] Zhang J, Yang H, Shen G, Cheng P, Zhang J, Guo S. Reduction of graphene oxide via L-ascorbic acid. Chem Comm 2010; 46: 1112-4.

[20] Dua V, Surwade SP, Ammu S, Agnihotra SR, Jain S, Roberts KE, et al. Allorganic vapor sensor using inkjet-printed reduced graphene oxide. Angew Chem Int Ed 2010; 49: 2154-7.

[21] Gao J, Liu F, Liu Y, Ma N, Wang Z, Zhang X. Environment-friendly method to produce graphene that employs vitamin C and amino acid. Chem Mater 2010; 22: 22138.

[22] Fernández-Merino MJ, Guardia L, Paredes JI, Villar-Rodil S, Solís-Fernández P, Martínez-Alonso A, et al. Vitamin C is an ideal substitute for hydrazine in the reduction of graphene oxide suspensions. J Phys Chem C 2010; 114: 6426-32. 
[23] Zhu C, Guo S, Fang Y, Dong S. Reducing sugar: New functional molecules for the green synthesis of graphene nanosheets. ACS Nano 2010; 4: 2429-37.

[24] Kim Y-K, Kim M-H, Min D-H. Biocompatible reduced graphene oxide prepared by using dextran as a multifunctional reducing agent. Chem Commun 2011; 47: 3195-7. [25] Akhavan O, Ghaderi E, Aghayee S, Fereydooni Y, Talebi A. The use of a glucosereduced graphene oxide suspension for photothermal cancer therapy. J. Mater Chem 2012; 22: 13773-81.

[26] Wang Y, Shi ZX, Yin J. Facile synthesis of soluble graphene via a green reduction of graphene oxide in tea solution and its biocomposites. ACS Appl Mater Interfaces 2011; 3: 1127-33.

[27] Lei Y, Tang Z, Liao R, Guo B. Hydrolysable tannin as environmentally friendly reducer and stabilizer for graphene oxide. Green Chem 2011; 13: 1655-8.

[28] Akhavan O, Kalaee M, Alavi ZS, Ghiasi SMA, Esfandiar A Increasing the antioxidant activity of green tea polyphenols in the presence of iron for the reduction of graphene oxide. Carbon 2012; 50: 3015-25.

[29] Wang Y, Shi ZX, Fan J, Ge Y, Yin J, Hub G. Self-assembly of graphene into threedimensional structures promoted by natural phenolic acids. J Mater Chem 2012; 22: 22459-66.

[30] Li J, Xiao G, Chen C, Li R, Yan D. Superior dispersions of reduced graphene oxide synthesized by using gallic acid as a reductant and stabilizer. J Mater Chem A 2013; 1: 1481-7.

[31] Mhamane D, Ramadan W, Fawzy M, Rana A, Dubey M, Rode C, et al. From graphite oxide to highly water dispersible functionalized graphene by single step plant extract-induced deoxygenation. Green Chem 2011; 13: 1990-6. 
[32] Kang SM, Park S, Kim D, Park SY, Ruoff RS, Lee H. Simultaneous reduction and surface functionalization of graphene oxide by mussel-inspired chemistry. Adv Func Mater 2011; 21: 108-12.

[33] Esfandiar A, Akhavan O, Irajizad A. Melatonin as a powerful bio-antioxidant for reduction of graphene oxide. J Mater Chem 2011; 21: 10907-14.

[34] Akhavan O, Ghaderi E, Esfandiar A. Wrapping bacteria by graphene nanosheets for isolation from environment, reactivation by sonication, and inactivation by Nearinfrared irradiation. J Phys Chem B 2011; 115: 6279-88.

[35] Pham TA, Kim JS, Kim JS, Jeong YT. One-step reduction of graphene oxide with L-glutathione. Colloid Surf A 2011; 384: 543-8.

[36] Liu J, Fu S, Yuan BM, Li Y, Deng Z. Toward a universal "adhesive nanosheet” for the assembly of multiple nanoparticles based on a protein-induced reduction/decoration of graphene oxide. J Am Chem Soc 2010; 132: 7279-81.

[37] Salas EC, Sun Z, Lüttge A, Tour JM. Reduction of graphene oxide via bacterial respiration. ACS Nano 2010; 4: 4852-6.

[38] Akhavan O, Ghaderi E. Escherichia coli bacteria reduce graphene oxide to bactericidal graphene in a self-limiting manner. Carbon 2012; 50: 1853-60.

[39] Paredes JI, Villar-Rodil S, Solís-Fernández P, Martínez-Alonso A, Tascón JMD. Atomic force and scanning tunneling microscopy imaging of graphene nanosheets derived from graphite oxide. Langmuir 2009; 25: 5957-68.

[40] Stocker P, Lesgards J-F, Vidal N, Chalier F, Prost M. ESR study of a biological assay on whole blood: antioxidant efficiency of various vitamins. Biochim Biophys Acta 2003; 1621: 1-8. 
[41] Endo N, Nishiyama K, Otsuka A, Kanouchi H, Taga M, Oka T. Antioxidant activity of vitamin $\mathrm{B}_{6}$ delays homocysteine-induced atherosclerosis in rats. Br $\mathbf{J}$ Nutr 2006; 95: 1088-93.

[42] Toyosaki T, Mineshita TJ. Antioxidant effect of riboflavin in aqueous solution. Agric Food Chem 1989; 37: 286-9.

[43] Papadopoulos K, Triantis T, Dimotikali D, Nikokavouras J. Evaluation of food antioxidant activity by photostorage chemiluminescence. Anal Chim Acta 2001; 433: 263-8.

[44] Manikam VR, Cheong KY, Arazak KA. Chemical reduction methods for synthesizing Ag and Al nanoparticles and their respective nanoalloys. Mater Sci Eng B 2011; 176: 187-203.

[45] Babizhayev MA. Antioxidant activity of L-carnosine, a natural histidine-containing dipeptide in crystalline lens. Biochim Biophys Acta 1989; 1004: 363-71.

[46] Capitani CD, Carvalho ACL, Rivelli PD, Barros SBM, Castro IA. Evaluation of natural and synthetic compounds according to their antioxidant activity using a multivariate approach. Eur J Lipid Sci Technol 2009; 111: 1090-9.

[47] Xie J, Lee JY, Wang DIC, Ting YP. Silver nanoplates: from biological to biomimetic synthesis. ACS Nano 2007, 1, 429-39.

[48] Solís-Fernández P, Rozada R, Paredes JI, Villar-Rodil S, Fernández-Merino MJ, Guardia L, et al. Chemical and microscopic analysis of graphene prepared by different reduction degrees of graphene oxide. J Alloys Compd 2012; S536: S532-7.

[49] Zhang G, Sun S, Yang D, Dodelet J-P, Sacher E. The surface analytical characterization of carbon fibers functionalized by $\mathrm{H}_{2} \mathrm{SO}_{4} / \mathrm{HNO}_{3}$ treatment. Carbon 2008; 46: 196-205. 
[50] Dimiev AM, Alemany LB, Tour JM. Graphene oxide. Origin of acidity, its instability in water, and a new dynamic structural model. ACSNano 2013; 7: 576-88.

[51] Solís-Fernández P, Paredes JI, Villar-Rodil S, Martínez-Alonso A, Tascón JMD. Determining the thickness of chemically modified graphenes by scanning probe microscopy. Carbon 2010; 48: 2657-60.

[52] Greenwood NN, Earnshaw A. Chemistry of the Elements. Pergamon Press. Oxford; 1989: 598.

[53] Moon IK, Lee J, Ruoff RS, Lee H. Reduced graphene oxide by chemical graphitization. Nature Commun 2010; 1: 73.

[54] Pei S, Zhao J, Du J, Ren W, Cheng H-M. Direct reduction of graphene oxide films into highly conductive and flexible graphene films by hydrohalic acids. Carbon 2010; 48: 4466-74.

[55] Liao K-S, Mittal A, Bose S, Leighton C, Mkhoyan KA, Macosko CW. Aqueous only route toward graphene from graphite oxide. ACS Nano 2011; 5: 1253-8.

[56] Feldheim DL, Foss CA. Metal Nanoparticles: Synthesis, Characterization \& Applications. Marcel Dekker. New York; 2002: 13.

[57] Nair LS, Laurencin CT. J. Silver nanoparticles: synthesis and therapeutic applications. Biomed Nanotechnol 2007; 3: 301-16.

[58] Nadagouda MN, Varma RS. Green and controlled synthesis of gold and platinum nanomaterials using vitamin $\mathrm{B}_{2}$ : density-assisted self-assembly of nanospheres, wires and rods. Green Chem 2006; 8: 516-8.

[59] Rosi NL, Mirkin CA. Nanostructures in biodiagnostics. Chem Rev 2005; 105: 1547-62. 
[60] Kamat PV. Graphene-based nanoarchitectures. Anchoring semiconductor and metal nanoparticles on a two-dimensional carbon support. J Phys Chem Lett 2010; 1: $520-7$.

[61] Mao C-F, Vannice MA. Formaldehyde oxidation over Ag catalysts. J Catal 1995; 154: $230-44$.

[62] Panacek A, Kvitec L, Prucek R, Kolar M, Vecerova R, Pizurova N, et al. Silver colloid nanoparticles: synthesis, characterization, and their antibacterial activity. Phys Chem B 2006; 110: 16248-53.

[63] Hu B, Wang S-B, Wang K, Zhang M, Yu S-H. Microwave-assisted rapid facile "green" synthesis of uniform silver nanoparticles: self-assembly into multilayered films and their optical properties. J Phys Chem C 2008; 112: 11169-74.

[64] Roy B, Bairi P, Nandi AK. Selective colorimetric sensing of mercury(II) using turn off-turn on mechanism from riboflavin stabilized silver nanoparticles in aqueous medium. Analyst 2011; 136: 3605-7.

[65] Jacob JA, Naumov S, Mukherjee T, Kapoor S. Preparation, characterization, surface modification and redox reactions of silver nanoparticles in the presence of tryptophan. Coll Surf B 2011; 87: 498-504.

[66] Liu Z, Xing Z, Zu Y, Tan S, Zhao L, Zhou Z, et al. Synthesis and characterization of L-histidine capped silver nanoparticles. Mater Sci Eng C 2012; 32: 811-6.

[67] Evanoff Jr. DD, Chumanov G. Synthesis and optical properties of silver nanoparticles and arrays. ChemPhysChem 2005; 6: 1221-1231.

[68] Crist BV. Handbook of Monochromatic XPS Spectra. Vol 1. The Elements \& Native Oxides. XPS International LLC. California, USA; 2004:1-4. 
[69] Hervés P, Pérez-Lorenzo M, Liz-Marzán LM, Dzubiella J, Lu Y, Ballauff M. Catalysis by metallic nanoparticles in aqueous solution: model reactions. Chem Soc Rev 2012; 41: 5577-87.

[70] Lu Y, Mei Y, Ballauff M, Drechsler M. Thermosensitive core-shell particles as carrier systems for metallic nanoparticles. J Phys Chem B 2006; 110: 3930-7.

[71] Eising R, Signori AM, Fort S, Domingos JB. Development of catalytically active silver colloid nanoparticles stabilized by dextran. Langmuir 2011; 27: 11860-6.

[72] Signori AM, Santos KO, Eising R, Albuquerque BL, Giacomelli FC, Domingos JB. Formation of catalytic silver nanoparticles supported on branched polyethyleneimine derivatives. Langmuir 2010; 26: 17772-9.

[73] Zhang JT, Wei G, Keller TF, Gallagher H, Stötzel C, Müller FA, et al. Responsive hybrid polymeric/metallic nanoparticles for catalytic applications. Macromol Mater Eng 2010; 295: 1049-57.

[74] Harish S, Sabarinathan R, Joseph J, Phani KLN. Role of pH in the synthesis of 3aminopropyl trimethoxysilane stabilized colloidal gold/silver and their alloy sols and their application to catalysis. Mater Chem Phys 2011; 127: 203-7.

[75] Murugadoss A, Chattopadhyay A. A 'green' chitosan-silver nanoparticle composite as a heterogeneous as well as micro-heterogeneous catalyst. Nanotechnology 2008; 19: 015603/1-/9.

[76] Zhang P, Shao C, Zhang Z, Zhang M, Mu J, Guo Z, et al. In situ assembly of welldispersed Ag nanoparticles (AgNPs) on electrospun carbon nanofibers (CNFs) for catalytic reduction of 4-nitrophenol. Nanoscale 2011; 3: 3357-63. 
[77] Lu Y, Mei Y, Schrinner M, Ballauff M, Möller MW, Breu J. In situ formation of Ag nanoparticles in spherical polyacrylic acid brushes by UV irradiation. J Phys Chem C 2007; 111: 7676-81.

[78] Rashid MdH, Mandal TK. Synthesis and catalytic application of nanostructured silver dendrites. J Phys Chem C 2007; 111: 16750-60.

[79] Xu R, Wang D, Zhang J, Li Y. Shape-dependent catalytic activity of silver nanoparticles for the oxidation of styrene. Chem Asian J 2006; 1: 888-93. 
